\title{
Papel antiaterogênico do Resveratrol e seu efeito sobre a pressão arterial
}

\author{
Antiaterogenic role of Resveratrol and its effect on blood pressure \\ Papel antiaterogênico del Resveratrol y su efecto sobre la presión arterial
}

Recebido: 29/07/2021 | Revisado: 02/08/2021 | Aceito: 03/08/2021 | Publicado: 08/08/2021

\author{
Luís Guilherme da Silva Cavalcante \\ ORCID: https://orcid.org/0000-0002-9762-4770 \\ Universidade Estácio de Sá, Brasil \\ E-mail: guilherme.nutricionista28@gmail.com \\ Maricelia Costa Rodrigues Santana \\ ORCID: https://orcid.org/0000-0002-7631-9072 \\ Universidade Estácio de Sá, Brasil \\ E-mail: maricelia2rodrigues@gmail.com \\ Rayanne Ramos Lula \\ ORCID: https://orcid.org/0000-0001-5080-5833 \\ Universidade Estácio de Sá, Brasil \\ E-mail: lularayanneramos@gmail.com \\ Ana Paula Ramos Silva \\ ORCID: https://orcid.org/0000-0003-3765-1491 \\ Universidade Estácio de Sá, Brasil \\ E-mail: paulinha.ramos1523@gmail.com \\ Lorena Raíssa Nobre Dantas \\ ORCID: https://orcid.org/0000-0001-9710-2772 \\ Universidade Estácio de Sá, Brasil \\ E-mail: lorenaraissand@gmail.com \\ Hyanka Ferro Camello \\ ORCID: https://orcid.org/0000-0001-8490-8996 \\ Universidade Estácio de Sá, Brasil \\ E-mail: hyankaferro97@gmail.com \\ Amanda Cristine Ferreira dos Santos \\ ORCID: https://orcid.org/0000-0002-1979-6745 \\ Universidade Estácio de Sá, Brasil \\ E-mail: profaamandaferreira@gmail.com
}

\begin{abstract}
Resumo
O objetivo do presente estudo foi investigar na literatura a ação do resveratrol como um antiaterogênico bem como seu efeito sobre a pressão arterial. Foram utilizados artigos publicados em três bases de dados científicas: Pubmed, Literatura Latino-Americana e do Caribe em Ciências da Saúde (Lilacs) e Scientific Electronic Library Online (SciELO), sendo adotados como critérios de elegibilidade estudos observacionais de coorte, caso-controle e transversais referentes a temática, publicados entre os anos de 2015 e 2020, nos idiomas inglês e português. O resveratrol apresentou efeitos positivos no tratamento e prevenção da aterosclerose e no controle da pressão arterial, no entanto, embora os estudos apresentados tenham se mostrado satisfatórios quanto a ação do RSV sobre a redução do risco de aterosclerose e uma diminuição significativa dos níveis de PA, é válido ressaltar que a maioria das pesquisas foram realizadas em camundongos, o que o implica em uma incerteza a respeito de seus efeitos sobre a população humana. A administração de resveratrol se mostrou altamente benéfica à saúde cardiovascular, sobretudo na melhora da função endotelial, perfil lipídico e diminuição do estresse oxidativo, o que culminou em um poderoso aliado no combate e prevenção da aterosclerose e na redução dos níveis de pressão arterial.
\end{abstract}

Palavras-chave: Resveratrol; Aterosclerose; Hipertensão arterial.

\begin{abstract}
The aim of the present study was to investigate in the literature the action of resveratrol as an antiatherogenic as well as its effect on blood pressure. Articles published in three scientific databases were used: Pubmed, Latin American and Caribbean Literature in Health Sciences (Lilacs) and Scientific Electronic Library Online (SciELO), being adopted as eligibility criteria observational cohort studies, case-control and transversals referring to the theme, published between 2015 and 2020, in English and Portuguese. Resveratrol had positive effects in the treatment and prevention of atherosclerosis and in the control of blood pressure, however, although the studies presented have been shown to be satisfactory regarding the action of RSV on reducing the risk of atherosclerosis and a significant decrease in BP levels, It is worth noting that most of the researches were carried out in mice, which implies an uncertainty about its effects on the human population. Resveratrol administration proved to be highly beneficial to cardiovascular
\end{abstract}


health, especially in improving endothelial function, lipid profile and reducing oxidative stress, which culminated in a powerful ally in combating and preventing atherosclerosis and in reducing blood pressure levels.

Keywords: Resveratrol; Atherosclerosis; Hypertension.

\section{Resumen}

El objetivo del presente estudio fue investigar en la literatura la acción del resveratrol como antiaterogénico así como su efecto sobre la presión arterial. Se utilizaron artículos publicados en tres bases de datos científicas: Pubmed, Literatura Latinoamericana y del Caribe en Ciencias de la Salud (Lilacs) y Scientific Electronic Library Online (SciELO), siendo adoptados como criterios de elegibilidad estudios de cohortes observacionales, casos-controles y transversales referentes al tema, publicado entre 2015 y 2020, en inglés y portugués. El resveratrol tuvo efectos positivos en el tratamiento y prevención de la aterosclerosis y en el control de la presión arterial, sin embargo, aunque los estudios presentados han demostrado ser satisfactorios en cuanto a la acción del VSR en la reducción del riesgo de aterosclerosis y una disminución significativa de los niveles de PA, Cabe señalar que la mayoría de las investigaciones se realizaron en ratones, lo que implica una incertidumbre sobre sus efectos en la población humana. La administración de resveratrol demostró ser altamente beneficiosa para la salud cardiovascular, especialmente en la mejora de la función endotelial, el perfil lipídico y la reducción del estrés oxidativo, lo que culminó en un poderoso aliado para combatir y prevenir la aterosclerosis y reducir los niveles de presión arterial.

Palabras clave: Resveratrol; Aterosclerosis; Hipertensión.

\section{Introdução}

Os polifenóis, compostos químicos amplamente encontrados em alimentos de origem vegetal, têm sido alvos de grandes estudos quanto aos seus efeitos na redução do risco de doenças crônicas, em especial sobre os distúrbios cardiovasculares (Ladeira et al., 2019).

Dentre os mais estudados, o Resveratrol (RSV), componente encontrado principalmente na casca e sementes da Vitis vinífera L. (uva) vem ganhando destaque por sua ação cardioprotetora, sobretudo no que se refere à aterosclerose e à hipertensão arterial, através de uma série de mecanismos que vão desde uma melhora na função endotelial até uma inibição da agregação plaquetária (Figueira; Zanchett, 2019).

A origem da hipótese sobre os benefícios do RSV sobre a saúde cardiovascular deu-se através do conhecido "Paradoxo francês", no qual pesquisadores observaram que, mesmo seguindo uma dieta rica em colesterol, a incidência de eventos cardiovasculares entre os franceses era baixa, associando este fato, posteriormente, ao consumo frequente de vinho, uma das mais conhecidas fontes do polifenol (Vatavuk-Serrati et al., 2018).

Neste contexto, os distúrbios cardiovasculares, sobretudo a aterosclerose e a hipertensão arterial, configuram-se como a principal causa de morte em todo o mundo, atingindo somente no Brasil, aproximadamente 31,8\% da população, sendo responsáveis por uma notável parcela dos gastos públicos em internações (Massa; Duarte; Filho, 2017).

Sob essa perspectiva, o presente estudo teve por objetivo investigar na literatura a ação do RSV como um antiaterogênico bem como seu efeito sobre a pressão arterial (PA).

\section{Metodologia}

Trata-se de uma revisão integrativa da literatura baseada na análise de estudos referentes ao papel do RSV como um cardioprotetor, com foco no seu efeito sobre a patogênese de distúrbios cardiovasculares. Para tanto, utilizando-se como questão focal: “Quais os efeitos da suplementação de resveratrol sobre a Aterosclerose e Hipertensão arterial?”

Adiante, conduziu-se uma busca por artigos indexados nas bases de dados PubMed, Scielo e Science Direct, empregando-se os descritores "Resveratrol”, "Hipertensão arterial” e "Aterosclerose", previamente verificados nos Descritores em Ciências da Saúde (DeCS), disponível no portal da BVS, sendo conectados, posteriormente, aos operadores booleanos “AND", "OR" e "NOT" 
Foram adotados como critérios de elegibilidade estudos observacionais de coorte, caso-controle e transversais referentes a temática, publicados entre os anos de 2015 e 2020, nos idiomas inglês e português. Foram excluídos artigos de revisão, relatos de casos ou série de casos e cartas ao editor. Utilizou-se o gerenciador de referências Mendeley Desktop 1.19.8. para a eliminação de estudos duplicados.

A seleção dos estudos foi conduzida por meio da leitura de títulos e resumos, com posterior leitura dos artigos por inteiro para então incluí-los ou não, técnica sugerida por Marconi e Lakatos (2003). Por conseguinte, as referências dos artigos selecionados foram rastreadas com vista a inclusão de pesquisas de potencial interesse. Tais procedimentos foram executados por 4 pesquisadores de forma simultânea e independente. Aqueles estudos que houveram discordâncias foram discutidos em reunião os mesmos para uma avalição e consenso.

Por fim, para a síntese dos estudos selecionados, por meio de um protocolo elaborado pelos pesquisadores, foram extraídos os seguintes dados: Autor/ano, objetivo, metodologia e conclusão. A Figura 1 apresenta o fluxograma da seleção dos estudos em suas respectivas bases de dados, bem como a quantidade total de artigos encontrados em cada uma delas.

Figura 1: fluxograma da seleção de estudos em suas respectivas bases de dados.

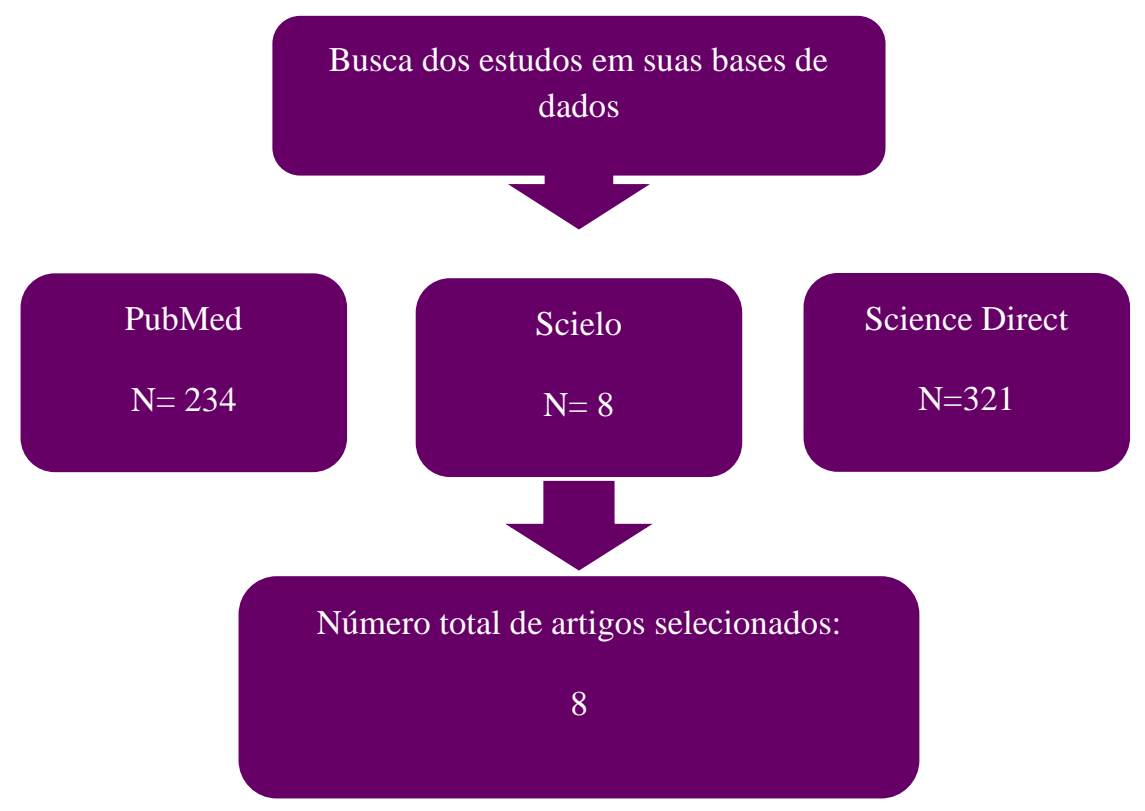

Fonte: Pesquisa direta.

\section{Resultados e Discussão}

Dos 7 estudos selecionados, 3 investigaram o papel do RSV quanto a atenuação dos eventos ateroscleróticos, enquanto que os quatro restantes se propuseram a devassar sua ação sobre a hipertensão arterial.

O Quadro 1 apresenta a síntese dos principais resultados de pesquisas referentes ao papel do RSV sobre a patogênese da aterosclerose. 
Quadro 1: Efeito antiaterogênico do RSV.

\begin{tabular}{|c|c|c|c|}
\hline Autor/Ano & Objetivo & Metodologia & Conclusão \\
\hline Chassot et al., 2018. & $\begin{array}{l}\text { Comparar o efeito do consumo } \\
\text { de vinho tinto com o consumo } \\
\text { de trans-resveratrol na } \\
\text { prevenção e regressão da } \\
\text { aterosclerose em } \\
\text { camundongos LDLr. }\end{array}$ & $\begin{array}{l}\text { Foram selecionados quatro } \\
\text { grupos, nos quais receberam } \\
\text { doses de vinho tinto e vinho } \\
\text { desalcoolizado, } 1 \text { com trans- } \\
\text { resveratrol e } 1 \text { grupo } \\
\text { controle. }\end{array}$ & $\begin{array}{l}\text { A suplementação, tanto com } \\
\text { o trans-resveratrol quanto } \\
\text { com os vinhos (tinto e sem } \\
\text { álcool) alterou } \\
\text { biomarcadores de estresse } \\
\text { oxidativo e lipidemia. }\end{array}$ \\
\hline Zhou et al., 2020 & $\begin{array}{c}\text { Observar os efeitos do } \\
\text { Resveratrol na aterosclerose } \\
\text { induzida por uma dieta rica } \\
\text { em lipídeos }\end{array}$ & $\begin{array}{l}\text { Foi submetido um grupo de } \\
\text { camundongos ApoE à uma } \\
\text { dieta rica em lipídeos, } \\
\text { administrando-se resveratrol } \\
\text { durante } 20 \text { semanas. }\end{array}$ & $\begin{array}{c}\text { A administração de } \\
\text { resveratrol atenuou a } \\
\text { aterosclerose induzida por } \\
\text { uma dieta rica em gorduras. }\end{array}$ \\
\hline Li et al., 2019. & $\begin{array}{l}\text { Investigar o efeito protetor do } \\
\text { Resveratrol na disfunção } \\
\text { endotelial induzida por uma } \\
\text { dieta rica em gordura. }\end{array}$ & $\begin{array}{l}\text { Células humanas foram } \\
\text { tratadas com resveratrol } \\
\text { enquanto camundongos } \\
\text { ApoE foram alimentados } \\
\text { com uma dieta rica em } \\
\text { lipídeos suplementada com } \\
\text { resveratrol por } 8 \text { semanas. }\end{array}$ & $\begin{array}{c}\text { o tratamento com resveratrol } \\
\text { melhorou a disfunção } \\
\text { endotelial e atenuou a } \\
\text { formação da placa de } \\
\text { ateroma nos camundongos } \\
\text { ApoE. }\end{array}$ \\
\hline Chang et al., 2015. & $\begin{array}{c}\text { Investigar os efeitos } \\
\text { antiaterogênicos do } \\
\text { Resveratrol em ratos com } \\
\text { deficiência de apolipoprerina e } \\
\text { com dieta rica em colesterol. }\end{array}$ & $\begin{array}{c}\text { Camundongos com } \\
\text { deficiência de } \\
\text { Apolipoproteína e } \\
\text { submetidos a uma dieta rica } \\
\text { em colesterol foram } \\
\text { submetidos a suplementação } \\
\text { de Resveratrol durante } 8 \\
\text { semanas. }\end{array}$ & $\begin{array}{c}\text { O Resveratrol preveniu a } \\
\text { progressão da aterosclerose } \\
\text { e reduziu os níveis de LDL- } \\
\text { C. }\end{array}$ \\
\hline
\end{tabular}

Fonte: Pesquisa direta.

A fisiopatologia da aterosclerose se dá, inicialmente, por uma lesão ao tecido do endotélio, no qual as lipoproteínas de baixa densidade passam a acumular-se no espaço subendotelial onde, posteriormente, sofrem oxidação, tornando-se favorável a eventos inflamatórios, principais responsáveis pelo processo aterogênico (Bertozzi et al., 2019).

Sob essa perspectiva, estudos observaram que a suplementação de RSV em camundongos induzidos à aterosclerose com dieta rica e lipídeos e colesterol diminuiu significativamente a proporção de células T CD4+, atenuando o estado inflamatório (ZHOU et al. 2020) além de uma redução notória da lipoproteína de baixa densidade (LDL-C), índice aterogênico (LDL-C/ HDL-C) e nos níveis de NF-kB, um marcador inflamatório cardiovascular (Chang et al., 2015).

Contudo, Chassot et al. (2019) demonstraram que a suplementação de trans-resveratrol e vinho tinto em camundongos submetidos a dieta aterogênica foi mais efetiva como protocolo de prevenção, ou seja, com administração anterior à alimentação hiperlipídica e, mesmo observando-se um redução significativa do colesterol total, o RSV não foi capaz de impedir a formação de estrias gordurosas ocasionadas pela dieta aterogênica sugerindo-se, desta forma, que os benefícios do polifenol apresentam melhores resultados quando associados à uma alimentação saudável.

Para mais, a suplementação de RSV reduziu relativamente os marcadores de lesão endotelial (VEGE e PCR) em um grupo de coelhos, o que por sua vez diminuiu expressivamente a formação de danos ateroscleróticos (Figueira; gonzález, 2018).

É perceptível que o efeito do RSV sobre a patogênese da aterosclerose está bem documentado, sobretudo no que se refere à atenuação de danos ao endotélio. Um estudo com células aórticas humanas (HAECs) demonstrou que o tratamento com RSV aumenta positivamente a expressão dos genes óxido nítrico sintase (eNOS) o que, por sua vez, influenciou em uma melhora da disfunção endotelial ocasionada pela aterosclerose (Li et al. 2019). 
No entanto, vale salientar que, nas pesquisas apresentadas, algumas doses administradas podem não ser facilmente alcançadas apenas em alimentos fontes deste composto bioativo, sendo possível encontrá-lo em maior abundância no vinho tinto, a depender ainda do tipo de uva utilizado e seu respectivo processo de fabricação (Leal et al., 2017).

O Quadro 2 apresenta a síntese dos achados quanto ao efeito do RSV sobre a PA.

Quadro 2: Efeito do RSV sobre os níveis de PA.

\begin{tabular}{|c|c|c|c|}
\hline Autor/Ano & Objetivo & Metodologia & Conclusão \\
\hline JIA et al., 2020. & $\begin{array}{c}\text { Estudar o efeito do resveratrol } \\
\text { na redução da pressão arterial } \\
\text { em um modelo de rato } \\
\text { grávida, }\end{array}$ & $\begin{array}{l}\text { Quarenta ratos fêmeas foram } \\
\text { divididos em } 4 \text { grupos nos } \\
\text { quais, dois foram submetidos à } \\
\text { administração de resveratrol, } \\
\text { sendo o } 1^{\circ} \text { com dieta } \\
\text { normossódica e o último com } \\
\text { acréscimo } 8 \% \text { de sal. }\end{array}$ & $\begin{array}{c}\text { O resveratrol diminuiu os } \\
\text { níveis de pressão arterial no } \\
\text { modelo de rato grávida } \\
\text { hipertensa. }\end{array}$ \\
\hline YEH et al., 2018. & $\begin{array}{c}\text { Investigar o efeito do } \\
\text { resveratrol na redução da } \\
\text { pressão arterial em ratos com } \\
\text { hipertensão induzida por } \\
\text { frutose. }\end{array}$ & $\begin{array}{l}\text { Os ratos foram submetidos à } \\
\text { uma dieta com } 10 \% \text { de frutose e } \\
10 \mathrm{mg} / \mathrm{kg} / \text { dia de resveratrol } \\
\text { durante } 1 \text { semana. }\end{array}$ & $\begin{array}{l}\text { A administração de } \\
\text { resveratrol diminuiu os } \\
\text { níveis de pressão arterial dos } \\
\text { ratos estudados. }\end{array}$ \\
\hline LI et al., 2016. & $\begin{array}{c}\text { Investigar a ação do } \\
\text { resveratrol na redução da } \\
\text { pressão arterial em ratos } \\
\text { espontaneamente hipertensos. }\end{array}$ & $\begin{array}{l}\text { Relaxamentos dependentes do } \\
\text { endotélio induzidos por } \\
\text { acetilcolina (Ach) em aortas de } \\
\text { ratos foram examinados em } \\
\text { câmaras orgânicas. A pressão } \\
\text { arterial foi determinada por } \\
\text { métodos de radiotelemetria. }\end{array}$ & $\begin{array}{l}\text { O resveratrol aumentou a } \\
\text { produção de óxido nítrico } \\
\text { melhorando a função } \\
\text { endotelial e reduziu a } \\
\text { pressão arterial em ratos } \\
\text { hipertensos. }\end{array}$ \\
\hline FABRÍCIO et al., 2017. & $\begin{array}{l}\text { Verificar o efeito de dois } \\
\text { períodos diferentes de } \\
\text { tratamento com resveratrol } \\
\text { sobre a pressão arterial em } \\
\text { ratas adultas } \\
\text { ovariectomizadas. }\end{array}$ & $\begin{array}{c}\text { Foram utilizadas ratas Wistar } \\
\text { com } 3 \text { meses de idade divididas } \\
\text { em grupos nos quais grupos } \\
\text { avoriectomizadas foram tratadas } \\
\text { com } 10 \mathrm{mg} / \mathrm{kg} / \text { dia de resveratrol } \\
\text { durante } 60-90 \text { dias. }\end{array}$ & $\begin{array}{c}\text { O tratamento com } \\
\text { resveratrol durante } 90 \text { dias é } \\
\text { capaz de diminuir a pressão } \\
\text { arterial em ratas } \\
\text { ovariectomizadas. }\end{array}$ \\
\hline
\end{tabular}

Fonte: Pesquisa direta.

O efeito do RSV sobre a redução da PA é resultante de três mecanismos, dos quais envolvem a diminuição do estresse oxidativo, elevando a liberação de óxido nítrico (NO), atenuação da oxidação gerada pela presença de radicais livres e uma elevação do status de proteínas atuantes na via do NO, na qual interferências negativas estão diretamente ligadas à uma lesão da função endotelial, muito comum em casos de hipertensão arterial (Albertoni; Schor, 2015).

Neste contexto, estudos demonstraram que a suplementação com RSV em camundongos induzidos a hipertensão arterial por meio de dietas ricas em sódio proporcionou um aumento da produção de NO e da excreção urinária de sódio o que, por consequência, reduziu significativamente os níveis de PA (Jia et al., 2020), além de atenuar expressivamente a geração de espécies reativas de oxigênio (EROS), enfatizando-se que o tratamento com RSV, realizado precocemente, atenua o estrese oxidativo e o risco de hipertensão arterial (Yea et al., 2018).

Não obstante, uma análise realizada por Aydin et al. (2017) mostrou que a administração de RSV em um grupo de ratos submetidos a um inibidor de NO (L-arginina), não foi capaz de evitar ou atenuar o aumento da PA. Porém em virtude de a pesquisa ter sido realizada em um grupo relativamente pequeno, os resultados desta podem ter sofrido limitações. Neste contexto, uma análise da função endotelial da aorta de ratos mostrou que o tratamento com RSV ocasionou uma redução da PA, ligando tais benefícios à produção de NO, reforçando mais uma vez o papel deste mecanismo como intermédio da ação do composto sobre as cardiopatias ( $\mathrm{Li}$ et al. 2016). 
Atualmente pesquisas têm demonstrado que este polifenol pode apresentar ainda resultados satisfatórios quanto a prevenção da hipertensão arterial advinda de outras patologias, como o Diabetes Mellitus (DM), por exemplo. Sob esse ponto de vista, um estudo realizado por Imamura et al. (2017), investigou o efeito da suplementação de RSV sobre os níveis de PA em portadores de DM tipo 2. Ao fim, constatou-se que os indivíduos suplementados obtiveram uma redução expressiva na PA sistólica, o que por sua vez, entra em concordância com os resultados dos estudos do quadro 2 , dos quais, mesmo realizados em grupos diferentes, apresentaram resultados semelhantes.

Ademais, a suplementação de RSV normalizou os níveis de PA e atenuou a disfunção endotelial em ratas, após um procedimento de ovariectomia. No entanto, diferentemente dos estudos anteriores, concomitante a estes resultados, curiosamente não foram identificadas diferenças na concentração sérica de NO, obtendo tais efeitos de forma independente deste mecanismo, o que culmina em um viés para investigações futuras (Fabrício et al., 2017).

Embora os estudos apresentados tenham se mostrado satisfatórios quanto a ação do RSV sobre a redução do risco de aterosclerose e uma diminuição significativa dos níveis de PA, é válido ressaltar que a maioria das pesquisas foram realizadas em camundongos, o que o implica em uma incerteza a respeito de seus efeitos sobre a população humana. Além do mais, os mecanismos pelos quais o RSV desempenha suas funções ainda não estão totalmente esclarecidos. Desse modo, são necessários maiores estudos em seres humanos acerca do seu êxito sobre a saúde cardiovascular bem como sua respectiva dose para a execução de tais ações.

\section{Conclusão}

A administração de RSV, de forma rotineira, mostrou-se altamente benéfica à saúde cardiovascular, sobretudo na melhora da função endotelial, perfil lipídico e diminuição do estresse oxidativo, o que culminou em um poderoso aliado no combate e prevenção da aterosclerose e no controle da PA.

Desta forma, em virtude de tamanhos benefícios, o RSV pode exercer papel importante como um cardioprotetor a ser incluído no cotidiano da população. Contudo, estudos mais precisos e estabelecendo uma dose segura a humanos são necessários antes que o mesmo passe a ser utilizado como estratégia terapêutica para o tratamento ou prevenção de cardiopatias.

Em conclusão, espera-se que o presente estudo abra portas para maiores investigações acerca dos efeitos do RSV sobre a saúde humana, uma vez que este, nos últimos anos, tem se mostrado promissor na terapia de distúrbios cardiovasculares. Para tanto, sugere-se uma maior ênfase sobre pesquisas, tanto laboratoriais como em seres humanos envolvolvendo a ação deste polifenol para uma melhor compreensão de seus reais benefícios na prática clínica.

\section{Referências}

Albertoni, G., \& Schor, N. (2015). Resveratrol desempenha importante papel no mecanismo de proteção na doença renal-mini-revisão. Brazilian Journal of Nephrology, 37, 106-114. https://doi.org/10.5935/0101-2800.20150015

Aydin, M., Susam, I., Cabuk, A. K., Gungor, B., Akdur, A. S., Aksulu, H. E., \& Cabuk, G. (2017). Resveratrol did not alter blood pressure in rats with nitric oxide synthase-inhibited hypertension. Cardiovascular journal of Africa, 28(3), 141-146. https://doi.org/10.5830/CVJA-2016-069.

Bertozzi, H., Leite, R. S., Yoshida, E. H., Silva, R. A. H., \& Dos santos, N. S. (2019). Uso do resveratrol na prevenção da aterosclerose. Revista Saúde em Foco. 11, 373-381.

Chang, G. R., Chen, P. L., Hou, P. H., \& Mao, F. C. (2015). Resveratrol protects against diet-induced atherosclerosis by reducing low-density lipoprotein cholesterol and inhibiting inflammation in apolipoprotein E-deficient mice. Iranian journal of basic medical sciences, 18(11), 1063. https://doi.org/10.22038/IJBMS.2015.6043

Chassot, L. N., Scolaro, B., Roschel, G. G., Cogliati, B., Cavalcanti, M. F., Abdalla, D. S., \& Castro, I. A. (2018). Comparison between red wine and isolated trans-resveratrol on the prevention and regression of atherosclerosis in LDLr (-/-) mice. The Journal of nutritional biochemistry, 61, 48-55. https://doi.org/10.1016/j.jnutbio.2018.07.014. 
Research, Society and Development, v. 10, n. 10, e213101018873, 2021

(CC BY 4.0) | ISSN 2525-3409 | DOI: http://dx.doi.org/10.33448/rsd-v10i10.18873

Fabricio, V., Oishi, J. C., Biffe, B. G., Ruffoni, L. D. G., Silva, K. A. D., Nonaka, K. O., \& Rodrigues, G. J. (2017). Tratamento com Resveratrol Normaliza a Função Endotelial e a Pressão Arterial em Ratas Ovariectomizadas. Arquivos Brasileiros de Cardiologia, 108, 116-121. https://doi.org/ $10.5935 / \mathrm{abc} .20170012$

igueira, L., \& González, J. C. (2018). Effect of resveratrol on seric vascular endothelial growth factor concentrations during atherosclerosis. Clínica e Investigación en Arteriosclerosis (English Edition), 30(5), 209-216. https://doi.org/10.1016 / j.arteri.2018.04.003.

Figueira, T. B. N., \& Cechinel Zanchett, C. C. (2019). Resveratrol: papel nas doenças cardiovasculares. Revista Brasileira Multidisciplinar. 22.(2), 48-58.

Imamura, H., Yamaguchi, T., Nagayama, D., Saiki, A., Shirai, K., \& Tatsuno, I. (2017). Resveratrol melhora a rigidez arterial avaliada pelo índice vascular cardio-tornozelo em pacientes com diabetes mellitus tipo 2. International heart journal, 58 (4), 577-583. https://doi.org/10.1536/ihj.16-373.

Jia, X., Zhang, R., Guo, J., Yue, H., Liu, Q., Guo, L., \& Zhang, Q. (2020). Resveratrol supplementation prevents hypertension in hypertensive pregnant rats by increasing sodium excretion and serum nitric oxide level. International journal of hypertension, 2020. https://doi.org/10.1016/ j.arteri.2018.04.003.

Ladeira, C., Marques, A., Coelho, A., Paulos, E., Bellém, F., \& Almeida, A. (2020). Benefícios das fibras e polifenóis na diminuição dos níveis de colesterol: perceção da população Portuguesa. Saúde \& Tecnologia, (21), 18-25.

Leal, J. B., Carvalho, F. O., Gonçalves, D. C., Leal, J. B., Da Silva, G. C. L., Carnevalli, L. C., \& Hoefel, A. L. (2017). Resveratrol: composição química e seus benefícios à saúde. RBONE-Revista Brasileira De Obesidade, Nutrição E Emagrecimento, 11(67), 620-629.

Li, J., Zhong, Z., Yuan, J., Chen, X., Huang, Z., \& Wu, Z. (2019). Resveratrol improves endothelial dysfunction and attenuates atherogenesis in apolipoprotein E-deficient mice. The Journal of nutritional biochemistry, 67, 63-71. https://doi.org/10.1016 / j.jnutbio.2019.01.022.

Li, X., Dai, Y., Yan, S., Shi, Y., Li, J., Liu, J., \& Mu, J. (2016). Resveratrol lowers blood pressure in spontaneously hypertensive rats via calcium-dependent endothelial NO production. Clinical and Experimental Hypertension, 38(3), 287-293. https://doi.org/10.3109 / 10641963.2015.1089882.

Marconi, M. D. A., \& Lakatos, E. M. (2003). Fundamentos de metodologia científica. Atlas.

Massa, K. H. C., Duarte, Y. A. O., \& Chiavegatto Filho, A. D. P. (2019). Análise da prevalência de doenças cardiovasculares e fatores associados em idosos, 2000-2010. Ciência \& Saúde Coletiva, 24, 105-114. https://doi.org/10.1590/1413-81232018241.02072017

Vatavuk-Serrati, G., Alves, R. T., Costa, E. S., Castro, A. G. P. D., \& Machado, V. A. (2019). Efeitos da suplementação de resveratrol sobre fatores de risco cardiovascular. Rev. Soc. Cardiol. Estado de Säo Paulo, 88-93.

Yeh, T. C., Shin, C. S., Chen, H. H., Lai, C. C., Sun, G. C., Tseng, C. J., \& Cheng, P. W. (2018). Resveratrol regulates blood pressure by enhancing AMPK signaling to downregulate a Rac1-derived NADPH oxidase in the central nervous system. Journal of Applied Physiology, 125(1), 40-48. https://doi.org/10.1152/ japplphysiol.00686.2017.

Yeh, T. C., Shin, C. S., Chen, H. H., Lai, C. C., Sun, G. C., Tseng, C. J., \& Cheng, P. W. (2018). Resveratrol regulates blood pressure by enhancing AMPK signaling to downregulate a Rac1-derived NADPH oxidase in the central nervous system. Journal of Applied Physiology, 125(1), 40-48. https://doi.org/10.1152 / japplphysiol.00686.2017. 